\title{
ODD AND EVEN BEHAVIOR WITH LSUB $m$ APPROXIMATION LEVEL IN HIGH-ORDER COUPLED CLUSTER METHOD (CCM) CALCULATIONS
}

\author{
D. J. J. FARNELL \\ Academic Department of Radiation Oncology, Division of Cancer Studies, \\ Faculty of Medical and Human Science, University of Manchester, \\ c/o Christie Hospital NHS Foundation Trust, Manchester M20 4BX, United Kingdom \\ damian.farnell@manchester.ac.uk

\section{R. F. BISHOP} \\ School of Physics and Astronomy, Schuster Building, \\ The University of Manchester, Manchester M13 9PL, United Kingdom
}

Received 23 April 2008

\begin{abstract}
The coupled cluster method (CCM) is a powerful and widely applied technique of modern-day quantum many-body theory. It has been used with great success in order to understand the properties of quantum magnets at zero temperature. This is largely due to the application of computational techniques that allow the method to be applied to high orders of approximation using a localized scheme known as the LSUB $m$ scheme. A hitherto unreported aspect of this scheme is that results for LSUB $m$ expectation values behave in distinctly different ways with odd and even values of $m$. Here, we consider the behavior of ground-state expectation values of odd and even orders of the CCM LSUBm approximation for unfrustrated spin-half Heisenberg antiferromagnets on the square and honeycomb lattice and the frustrated spin-half Heisenberg antiferromagnet on the triangular lattice. We demonstrate that results for odd and even orders of approximation show qualitatively different behavior for both the ground-state energy and the sublattice magnetization. Indeed, the odd series consistently forms an upper branch of results, and the even series a lower branch with respect to both ground-state energy and sublattice magnetization, for all of the models considered here.
\end{abstract}

Keywords: Quantum magnetism; coupled cluster method; computational simulation.

\section{Introduction}

The coupled cluster method $(\mathrm{CCM})^{1-13}$ is a well-known method of quantum manybody theory (QMBT). The CCM has been applied with much success over the last 15 years or so in order to study quantum magnetic systems at zero temperature (see Refs. 14-43). In particular, the use of computer-algebraic implementations ${ }^{27,30,35}$ of the CCM for quantum systems of large or infinite numbers of particles has largely been found to be very effective with respect to these spin-lattice problems. This approach uses a localized approximation known as the LSUB $m$ approximation. 
The extent of the locale over which multi-spin correlations are explicitly included in the approximation is defined by the index $m$, and ground- and excited-state expectation values are extrapolated to the limit $m \rightarrow \infty$.

We apply the CCM to the spin-half Heisenberg model on the square, honeycomb, and triangular lattices. The Hamiltonian is specified as follows,

$$
H=\sum_{\langle i, j\rangle} \mathbf{s}_{i} \cdot \mathbf{s}_{j},
$$

where the sum on $\langle i, j\rangle$ counts all nearest-neighbor pairs once. The ground states of all of the cases considered here are classically ordered, albeit by a reduced amount due to quantum fluctuations. Indeed, the best estimates of the amount of classical ordering of the square lattice case from approximate methods are $62 \%$ from CCM ${ }^{30} 61.4 \%$ from quantum Monte Carlo studies, ${ }^{44} 61.4 \%$ from series expansions, ${ }^{45} 61.38 \%$ from spin-wave theory, ${ }^{46}$ and $63.4 \%$ from exact diagonalizations. ${ }^{47}$ For the honeycomb lattice case, the best estimates are $47 \%$ from quantum Monte Carlo studies, ${ }^{48} 53 \%$ from series expansions, ${ }^{49} 56 \%$ from exact diagonalizations, ${ }^{47}$ and $56 \%$ from previous CCM calculations. ${ }^{32}$ By contrast, the best estimates of the amount of classical ordering for the triangular lattice antiferromagnet are $41 \%$ from quantum Monte Carlo studies, ${ }^{50} 40 \%$ from series expansions, ${ }^{51} 39 \%$ from exact diagonalizations, ${ }^{47}$ and $52 \%$ from previous CCM calculations. ${ }^{33}$ In this article, we investigate the effects of considering odd and even values of $m$ on high-order CCM results.

\section{Method}

\subsection{The CCM formalism}

A description of the normal coupled cluster method (NCCM) formalism is given in Refs. 1-43. We note here simply that the exact ket and bra ground-state energy eigenvectors, $|\Psi\rangle$ and $\langle\tilde{\Psi}|$, of a general many-body system described by a Hamiltonian $H$

$$
H|\Psi\rangle=E_{g}|\Psi\rangle ; \quad\langle\tilde{\Psi}| H=E_{g}\langle\tilde{\Psi}|,
$$

are parametrized within the single-reference CCM as follows:

$$
\begin{gathered}
|\Psi\rangle=\mathrm{e}^{S}|\Phi\rangle ; \quad S=\sum_{I \neq 0} \mathcal{S}_{I} C_{I}^{+}, \\
\langle\tilde{\Psi}|=\langle\Phi| \tilde{S} \mathrm{e}^{-S} ; \quad \tilde{S}=1+\sum_{I \neq 0} \tilde{\mathcal{S}}_{I} C_{I}^{-} .
\end{gathered}
$$

Note that $|\Phi\rangle$ is the normalized single model or reference state. The correlation operator $S$ is thus a linked-cluster operator that is decomposed in Eq. (3) in terms of a complete set of mutually commuting multi-spin and multi-configurational creation operators $C_{I}^{+}$with respect to the model state. The hermitian adjoints, $C_{I}^{-}=\left(C_{I}^{+}\right)^{\dagger}$ are the corresponding destruction operators, $\left\langle\Phi\left|C_{I}^{+}=0=C_{I}^{-}\right| \Phi\right\rangle$, and we explicitly 
define $C_{0}^{+}=1$, the identity operator. The label $I$ is thus a set-index comprising a set of single-particle labels in some suitable single-particle basis defined via $|\Phi\rangle$. We note that the normalization conditions $\langle\Phi \mid \Psi\rangle=\langle\Phi \mid \Phi\rangle=1=\langle\tilde{\Psi} \mid \Psi\rangle$ follow from Eq. (3). The ground ket- and bra-state equations are given in terms of $\bar{H}=\langle\tilde{\Psi}|H| \Psi\rangle$ as

$$
\begin{aligned}
& \delta \bar{H} / \delta \tilde{\mathcal{S}}_{I}=0 \Rightarrow\left\langle\Phi\left|C_{I}^{-} \mathrm{e}^{-S} H \mathrm{e}^{S}\right| \Phi\right\rangle=0, \quad \forall I \neq 0 ; \\
& \delta \bar{H} / \delta \mathcal{S}_{I}=0 \Rightarrow\left\langle\Phi\left|\tilde{S} \mathrm{e}^{-S}\left[H, C_{I}^{+}\right] \mathrm{e}^{S}\right| \Phi\right\rangle=0, \quad \forall I \neq 0 .
\end{aligned}
$$

In order to solve for the Heisenberg model on the square, honeycomb and triangular lattices, we make approximations in both $S$ and $\tilde{S}$. The three most commonly employed approximation schemes previously utilized have been: (1) the SUB $n$ scheme, in which all correlations involving only $n$ or fewer spins are retained, but no further restriction is made concerning their spatial separation on the lattice; (2) the $\mathrm{SUB} n-m$ sub-approximation, in which all $\mathrm{SUB} n$ correlations spanning a range of no more than $m$ adjacent lattice sites are retained; and (3) the localized LSUB $m$ scheme, in which all multi-spin correlations over all distinct locales on the lattice defined by $m$ or fewer contiguous sites are retained. Equation (4) shows that the ground-state energy at the stationary point has the simple form

$$
E_{g}=E_{g}\left(\left\{\mathcal{S}_{I}\right\}\right)=\left\langle\Phi\left|\mathrm{e}^{-S} H \mathrm{e}^{S}\right| \Phi\right\rangle .
$$

Here, we use the classical ground state as the model state: for the square and honeycomb lattices, where this is a state in which neighboring spins are antiparallel; and, for the triangular lattice, we use a model state in which spins on three sublattices are at $120^{\circ}$ to each other. We also find the sublattice magnetization with respect to the bipartite square and honeycomb lattices and the tripartite triangular lattice. The CCM calculations were carried out to high-order using a computational code written by us. ${ }^{53}$ Furthermore, the specific details of setting up the CCM calculations for the square, honeycomb and triangular lattice antiferromagnets have been discussed at length in Refs. 29 and 27, respectively, and so are not discussed here further. Again, we note that the details of applying the CCM to a range of lattice quantum spin systems are given in Refs. 14-43.

\subsection{Extrapolation of CCM expectation values}

The aim of the present study is not to find new ways to extrapolate the LSUBm data. Rather, it is to employ a simple approach that has been used many times before to extrapolate CCM LSUB $m$ data in order to investigate the odd/even behavior with $m$. Thus, we note that results of LSUB $m$ calculations were extrapolated to the limit $m \rightarrow \infty$ by carrying out a quadratic fit of this data via least-squares estimation with respect to either $m^{-2}$ for the ground-state energy or $m^{-1}$ for the sublattice magnetization. The interested reader is referred to Ref. 30 in order to see how CCM expectation values may be extrapolated by using a "power-law" or by 
using Padé approximants. As is usual in CCM calculations, LSUB2 data is omitted from these extrapolations as they are rather far from the $m \rightarrow \infty$ limit and hence conform poorly to any type of extrapolation scheme (see, e.g., Refs. 35 and 43).

\section{Results}

The results for the ground-state energy for the square lattice are shown in Fig. 1 and in Table 1. We see a strong difference in the results for odd and even values of

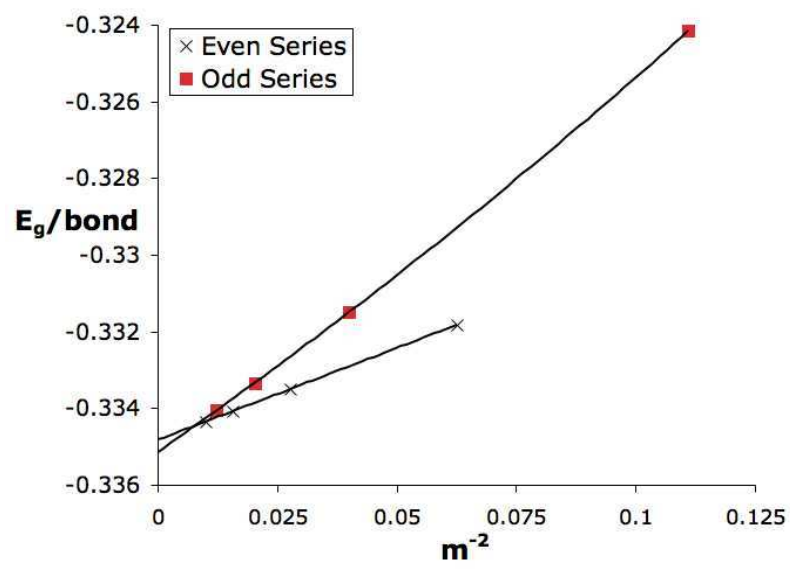

Fig. 1. CCM results for the ground-state energy of the spin-half Heisenberg antiferromagnet on the square lattice. Odd $(m=\{3,5,7,9\})$ and even $(m=\{4,6,8,10\})$ series of $\operatorname{LSUB} m$ results with respect to $m$ are plotted separately. The solid lines are fits of quadratic functions to the odd and even data.

Table 1. CCM results for ground-state energies per bond of the spin-half Heisenberg antiferromagnet for the square, honeycomb and triangular lattices compared to results of other methods.

\begin{tabular}{llll}
\hline & \multicolumn{1}{c}{ Square } & \multicolumn{1}{c}{ Honeycomb } & \multicolumn{1}{c}{ Triangle } \\
\hline LSUB3 & -0.324166 & -0.35 & -0.1730360 \\
LSUB4 & -0.331832 & -0.360143 & -0.1780889 \\
LSUB5 & -0.331496 & -0.359014 & -0.1795632 \\
LSUB6 & -0.333500 & -0.361848 & -0.1809668 \\
LSUB7 & -0.333360 & -0.361278 & -0.1816737 \\
LSUB8 & -0.334087 & -0.362434 & -0.1822621 \\
LSUB9 & -0.334042 & -0.362134 & - \\
LSUB10 & -0.334513 & -0.362688 & - \\
Odd Series Extrapolation & -0.335145 & -0.363473 & -0.184016 \\
Even Series Extrapolation & -0.334831 & -0.363155 & -0.184147 \\
Spin-Wave Theory & -0.334995 (Ref. 46) & -0.365929 (Ref. 49) & -0.1823 (Ref. 52) \\
Quantum Monte Carlo & $-0.334719(3)$ (Ref. 44) & -0.3630 (Ref. 48) & -0.1819 (Ref. 50) \\
Series Expansions & $-0.33465(5)$ (Ref. 45) & -0.3629 (Ref. 49) & -0.1842 (Ref. 51) \\
Exact Diagonalizations (Ref. 47) & -0.3350 & -0.3632 & -0.1842 \\
Previous CCM Results & -0.33485 (Ref. 30) & -0.3631 (Ref. 32) & -0.1837 (Ref. 33) \\
\hline
\end{tabular}


$m$ for the LSUB $m$ approximation level. Indeed, we find that the odd- $m$ results form an upper branch of results for the ground-state energy, whereas the even- $m$ results form a lower branch. Odd $(m=\{3,5,7,9\})$ and even $(m=\{4,6,8,10\})$ series of $\mathrm{LSUB} m$ results are extrapolated separately with respect to $m^{-2}$ in order to obtain approximate results in the limit $m \rightarrow \infty$. We see in Table 1 that the extrapolated values for the odd and even data appear to agree well with each other, although naturally, they are not identical.

Furthermore, as may be seen in Table 1, the CCM results for the ground-state energy also compare very favorably to those results of other methods and previous CCM extrapolations. For example, they compare well with previous CCM extrapolations that were carried out only on even values of $m$ for the ground-state energy of the spin-half square lattice. The extrapolated values of both the odd and even series of results for the ground-state energy fall very slightly below the results of the series expansions ${ }^{45}$ and quantum Monte Carlo ${ }^{44}$ techniques, although not that of spin-wave theory. ${ }^{46}$

Figure 2 and Table 2 indicate that the results for the LSUB $m$ sublattice magnetization for the square antiferromagnet also show similar odd/even behavior with approximation index $m$. Again, the odd series forms an upper branch of results and the even series forms a lower branch. Odd $(m=\{3,5,7,9\})$ and even $(m=\{4,6,8,10\})$ series of LSUB $m$ results are extrapolated with respect to $m^{-1}$. The odd and even extrapolation results again agree very well with each other. These results also compare well with the results of other methods, such as quantum Monte Carlo, ${ }^{44}$ series expansions, ${ }^{45}$ and spin-wave theory, ${ }^{46}$ as shown in Table 2.

The results for the ground-state energy and sublattice magnetizations of the honeycomb lattice spin-half antiferromagnet are presented in Figs. 3 and 4 and in

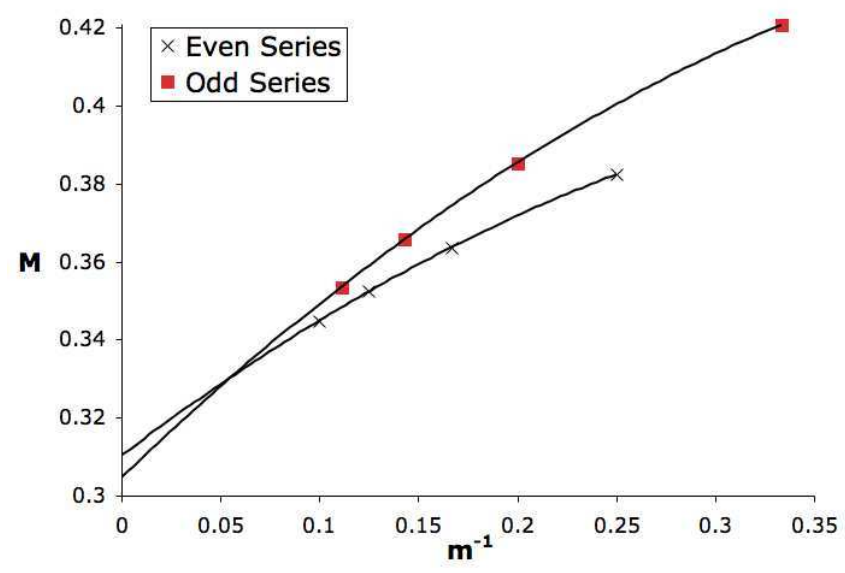

Fig. 2. CCM results for the sublattice magnetization of the spin-half Heisenberg antiferromagnet on the square lattice. Odd $(m=\{3,5,7,9\})$ and even $(m=\{4,6,8,10\})$ series of $\operatorname{LSUB} m$ results with respect to $m$ are plotted separately. The solid lines are fits of quadratic functions to the odd and even data. 
Table 2. CCM results for sublattice magnetizations of the spin-half Heisenberg antiferromagnet for the square, honeycomb and triangular lattices compared to results of other methods.

\begin{tabular}{llll}
\hline & \multicolumn{1}{c}{ Square } & \multicolumn{1}{c}{ Honeycomb } & \multicolumn{1}{c}{ Triangle } \\
\hline LSUB3 & 0.420713 & 0.4 & 0.402272 \\
LSUB4 & 0.382398 & 0.352173 & 0.363658 \\
LSUB5 & 0.385298 & 0.360373 & 0.347918 \\
LSUB6 & 0.363641 & 0.331345 & 0.328034 \\
LSUB7 & 0.365824 & 0.339114 & 0.315171 \\
LSUB8 & 0.352417 & 0.318995 & 0.301790 \\
LSUB9 & 0.353526 & 0.325449 & - \\
LSUB10 & 0.344833 & 0.310935 & - \\
Odd Series Extrapolation & 0.304656 & 0.272283 & 0.208489 \\
Even Series Extrapolation & 0.310243 & 0.274066 & 0.189331 \\
Spin-Wave Theory & 0.3069 (Ref. 46) & 0.2418 (Ref. 49) & 0.2387 (Ref. 52) \\
Quantum Monte Carlo & $0.3070(3)$ (Ref. 44) & 0.235 (Ref. 48) & 0.205 (Ref. 50) \\
Series Expansions & $0.307(1)$ (Ref. 45) & 0.266 (Ref. 49) & 0.20 (Ref. 51) \\
Exact Diagonalizations (Ref. 47) & 0.3173 & 0.2788 & 0.193 \\
Previous CCM Results & 0.31 (Ref. 30) & 0.28 (Ref. 32) & 0.258 (Ref. 33) \\
\hline
\end{tabular}

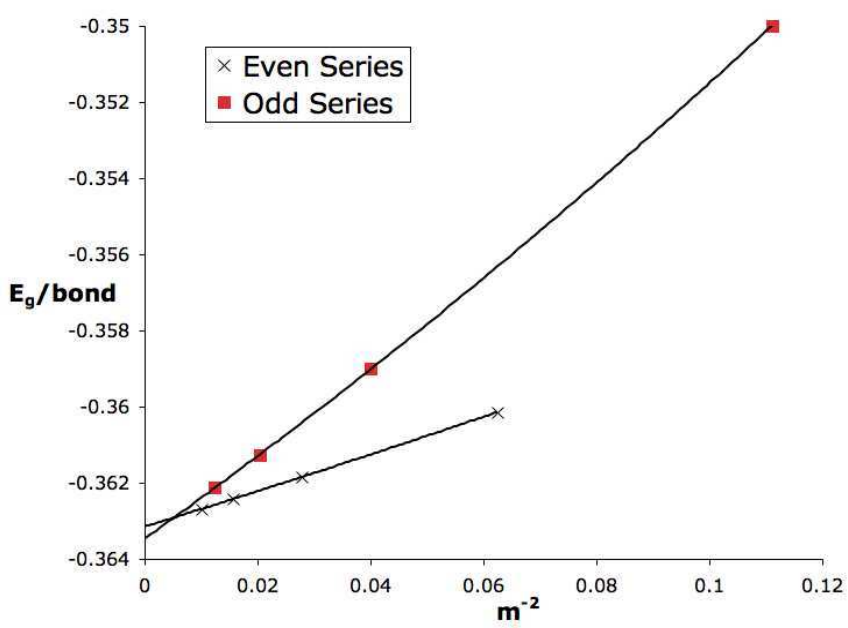

Fig. 3. CCM results for the ground-state energy of the spin-half Heisenberg antiferromagnet on the honeycomb lattice. Odd $(m=\{3,5,7,9\})$ and even $(m=\{4,6,8,10\})$ series of LSUB $m$ results with respect to $m$ are plotted separately. The solid lines are fits of quadratic functions to the odd and even data.

Tables 1 and 2. Again, we see the odd/even behavior with different levels of approximation level $m$, where the odd series forms the upper branch and the even series the lower. Odd $(m=\{3,5,7,9\})$ and even $(m=\{4,6,8,10\})$ series of LSUB $m$ results are extrapolated. The values for the extrapolated ground-state energy in Table 1 show that the odd and even extrapolations again compare well both with each other and with the best results of other methods, namely, those of series expansions ${ }^{51}$ and 


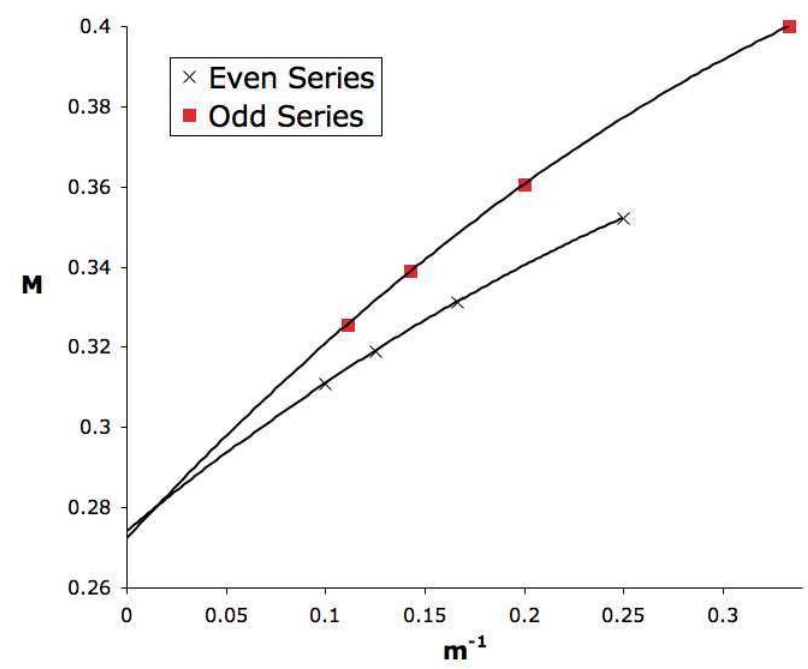

Fig. 4. CCM results for the sublattice magnetization of the spin-half Heisenberg antiferromagnet on the honeycomb lattice. Odd $(m=\{3,5,7,9\})$ and even $(m=\{4,6,8,10\})$ series of LSUB $m$ results with respect to $m$ are plotted separately. The solid lines are fits of quadratic functions to the odd and even data.

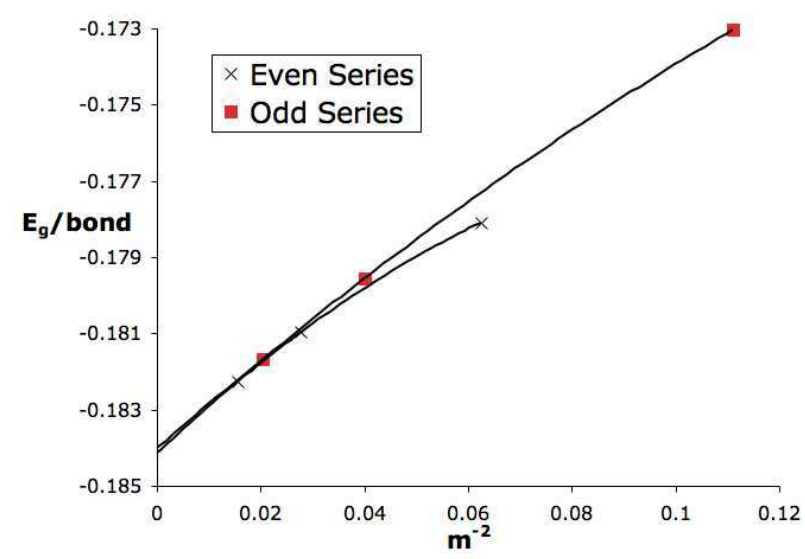

Fig. 5. CCM results for the ground-state energy of the spin-half Heisenberg antiferromagnet on the triangular lattice. Odd $(m=\{3,5,7\})$ and even $(m=\{4,6,8\})$ series of LSUB $m$ results with respect to $m$ are plotted separately. The solid lines are fits of quadratic functions to the odd and even data.

exact diagonalizations. ${ }^{47}$ Similarly, extrapolations of the sublattice magnetization also compare well with the results of other methods in Table 2, namely, quantum Monte Carlo, ${ }^{48}$ series expansions ${ }^{49}$ and exact diagonalizations. ${ }^{47}$

Figures 5 and 6 and Tables 1 and 2 present the results for the LSUB $m$ groundstate energy and the sublattice magnetization for the frustrated triangular lattice antiferromagnet. These results show similar odd/even behavior with approximation 


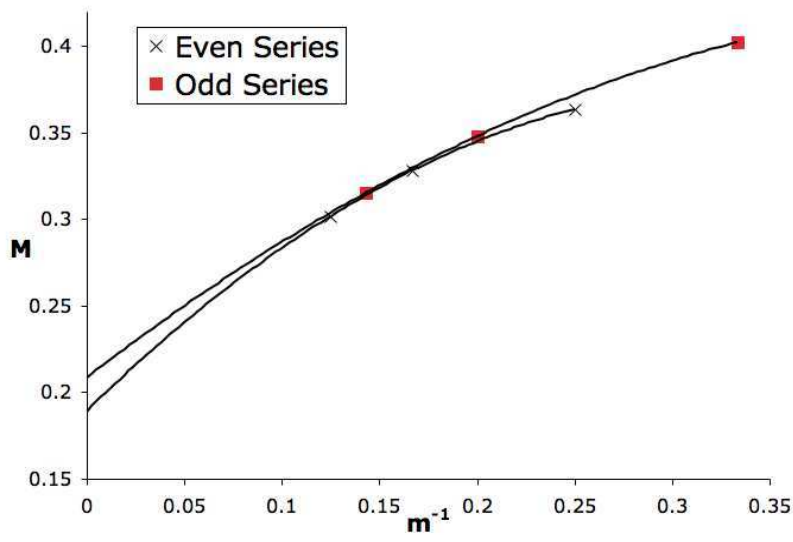

Fig. 6. CCM results for the sublattice magnetization of the spin-half Heisenberg antiferromagnet on the triangular lattice. Odd $(m=\{3,5,7\})$ and even $(m=\{4,6,8\})$ series of LSUB $m$ results with respect to $m$ are plotted separately. The solid lines are fits of quadratic functions to the odd and even data.

index $m$. Odd $(m=\{3,5,7\})$ and even $(m=\{4,6,8\})$ series of LSUB $m$ results are extrapolated. Extrapolated values for the ground-state energy per bond and sublattice magnetization are in good agreement with quantum Monte Carlo, ${ }^{50}$ series expansions, ${ }^{51}$ and exact diagonalizations. ${ }^{47}$

The previous extrapolated results for the $\mathrm{CCM}^{33}$ for the triangular lattice were carried out using all of the data (i.e., both odd and even series) due to the small amount of LSUBm data available at that time (i.e., up to LSUB6 only). This is due to the fact that the computational difficulty of the problem grows with the coordination number of the lattice. We note that the triangular lattice has a coordination number of 6 ; compared to a coordination number of 4 for the square lattice and 3 for the honeycomb lattice. Thus, we were only able to carry out high-order calculations here up to LSUB8 only for the triangular lattice because of this problem - even by using advanced parallel processing techniques. (We note that our current limit for high-order CCM via parallel processing is approximately $10^{5}$ fundamental clusters.) Our new results are also shown in Tables 1 and 2. The current CCM results for the sublattice magnetization, using only odd or only even extrapolated values of the sublattice magnetization, agree well with the results of quantum Monte Carlo, ${ }^{50}$ series expansions, ${ }^{51}$ and exact diagonalizations. ${ }^{47}$ The agreement is much better than previous CCM results ${ }^{33}$ that used all of the data (i.e., both odd and even orders up to LSUB6) available at that time.

\section{Conclusion}

We have considered the behavior of ground-state expectation values of odd and even orders of the CCM LSUBm approximation for unfrustrated spin-half Heisenberg antiferromagnets on the square and honeycomb lattice and the frustrated spin-half Heisenberg antiferromagnet on the triangular lattice. We showed that results for 
odd and even orders of approximation demonstrate qualitatively different behavior for both the ground-state energy and the sublattice magnetization. Indeed, the odd series consistently formed an upper branch of results, and the even series a lower branch with respect to both ground-state energy and sublattice magnetization, and for all of the models considered here. We believe that this behavior is interesting and is therefore note-worthy.

Indeed, odd/even behavior with $m$ has been observed by us in every lattice quantum spin problem ever considered that also has only two-spin interactions in the Hamiltonian, when treated by using high-order LSUB $m$ CCM techniques. It would be interesting to determine if similar properties with odd and even levels of approximation might also be seen if we looked at different types of expectation values, such as for the spin stiffness or for excitation energies. The only model to date that has not shown similar odd/even behavior was for the Ising model in a transverse field that contained both single-spin and double-spin terms in the Hamiltonian. ${ }^{31}$

The odd/even staggering behavior observed here is highly reminiscent of the behavior of expectation values evaluated directly at odd and even orders of perturbation theory (see, e.g., Ref. 54). Indeed, it is known ${ }^{27}$ that the CCM LSUBm approximation exactly reproduces perturbation theory to $m$ th order for quantum spin lattice systems. It is perhaps no surprise therefore that we also see such behavior with odd and even values of $m$ for the LSUB $m$ approximation. However, it should also be noted that the CCM LSUB $m$ scheme also does much more than simply reproduce $m$ th-order perturbation theory; many of the perturbation expansion coefficients with higher order than $m$ are approximated ${ }^{27}$ very well by the LSUB $m$ approximation, for example. Therefore, the analogy is not quite as straightforward as it first appears.

For the square and honeycomb lattices, we note that the total spin in the $z$ direction $\left(s_{T}^{z}=\sum_{i}^{N} s_{i}^{z}\right)$ is a conserved quantity, and so the list of fundamental configurations used in the CCM calculations contains only those clusters with even numbers of spin flips with respect to the Néel model state. We note that a similar effect with odd and even $m$ may be seen for LSUB $m$ calculations for the spinhalf linear chain Heisenberg antiferromagnet. In this case, $s_{T}^{z}$ is again a conserved quantity, and so only even numbers of spin flips are allowed in the list of fundamental configurations. We remember that the LSUB $m$ scheme allows all configurations in a locale defined by $m$ contiguous sites on the lattice. Hence, even though we may be restricted to even numbers of spin flips, these spin flips may be spread over an odd number of sites. For example, at order LSUB5 in one-dimension, there is a four-spin cluster that is spread over five adjacent sites, namely, at sites $(1,2,4,5)$. Clearly, similar situations also occur for two- or three-dimensional lattices when $m$ is an odd number. It is interesting to speculate that this might lie at the heart of the odd/even behavior for Hamiltonians with only two-spin interactions in them.

A limitation of the CCM method is that we have no known rules for extrapolation that can be rigorously justified on theoretical grounds. Rather, the rules that 
we have used here are heuristic in nature. This limitation is compounded by the fact that we may only have results for a small number of orders of approximation if we restrict our extrapolations to those results of only even or only odd series. For example, if we extrapolate LSUB2, LSUB4, and LSUB6 results using a quadratic function (as above) then there is no "error of extrapolation" as such. Indeed, we can generally perform an exact fit. Here, we extrapolated odd and even results for the ground-state energy and sublattice magnetization separately for the square, honeycomb, and triangular lattices. Although in principle these extrapolated values should agree precisely in the limit $m \rightarrow \infty$, they were found, in practice, to differ slightly. In the absence of any known rules for the behavior of the CCM expectation values with increasing LSUB $m$ approximation level, however, it is unlikely that the differing odd and even extrapolated values could be used to estimate the amount of error of extrapolation quantitatively.

Finally, the extrapolated values using only odd and only even series of data were both found to compare well with those results of the best of other methods for the ground-state energy and sublattice magnetization for the cases studied here. Indeed, our results were found to greatly improve previous results ${ }^{33}$ for the sublattice magnetization for the triangular lattice that utilized both odd and even values of $m$ together in order to extrapolate to the limit $m \rightarrow \infty$ due to the small number of data points available at that time. This indicates that our previous decision to extrapolate only even values (where possible) of LSUBm results (compared to extrapolating all of the odd and even data together) was a good choice.

\section{References}

1. F. Coester, Nucl. Phys. 7, 421 (1958).

2. F. Coester and H. Kümmel, Nucl. Phys. 17, 477 (1960).

3. J. Čižek, J. Chem. Phys. 45, 4256 (1966).

4. J. Čižek, Adv. Chem. Phys. 14, 35 (1969).

5. R. F. Bishop and K. H. Lührmann, Phys. Rev. B 17, 3757 (1978).

6. H. Kümmel, K. H. Lührmann and J. G. Zabolitzky, Phys. Rep. C 36, 1 (1978).

7. J. S. Arponen, Ann. Phys. (N.Y.) 151, 311 (1983).

8. R. F. Bishop and H. Kümmel, Phys. Today 40(3), 52 (1987).

9. J. S. Arponen, R. F. Bishop and E. Pajanne, Phys. Rev. A 36, 2519 (1987).

10. J. S. Arponen, R. F. Bishop and E. Pajanne, Phys. Rev. A 36, 2539 (1987).

11. J. S. Arponen, R. F. Bishop and E. Pajanne, in Condensed Matter Theories, Vol. 2, eds. P. Vashishta, R. K. Kalia and R. F. Bishop (Plenum, New York, 1987), p. 357.

12. R. J. Bartlett, J. Phys. Chem. 93, 1697 (1989).

13. R. F. Bishop, Theor. Chim. Acta 80, 95 (1991).

14. M. Roger and J. H. Hetherington, Phys. Rev. B 41, 200 (1990).

15. M. Roger and J. H. Hetherington, Europhys. Lett. 11, 255 (1990).

16. R. F. Bishop, J. B. Parkinson and Y. Xian, Phys. Rev. B 44, 9425 (1991).

17. R. F. Bishop, J. B. Parkinson and Y. Xian, Phys. Rev. B 46, 880 (1992).

18. R. F. Bishop, J. B. Parkinson and Y. Xian, J. Phys.: Condens. Matter 5, 9169 (1993).

19. D. J. J. Farnell and J. B. Parkinson, J. Phys.: Condens. Matter 6, 5521 (1994).

20. Y. Xian, J. Phys.: Condens. Matter 6, 5965 (1994). 
21. R. Bursill, G. A. Gehring, D. J. J. Farnell, J. B. Parkinson, T. Xiang and C. Zeng, J. Phys.: Condens. Matter 7, 8605 (1995).

22. R. Hale, High order coupled cluster method calculations for quantum spin systems, Ph.D. Thesis, UMIST, Manchester, United Kingdom (1995).

23. R. F. Bishop, R. G. Hale and Y. Xian, Phys. Rev. Lett. 73, 3157 (1994).

24. R. F. Bishop, D. J. J. Farnell and J. B. Parkinson, J. Phys.: Condens. Matter 8, 11153 (1996).

25. D. J. J. Farnell, S. A. Krüger and J. B. Parkinson, J. Phys.: Condens. Matter 9, 7601 (1997).

26. R. F. Bishop, Y. Xian and C. Zeng, in Condensed Matter Theories, Vol. 11, eds. E. V. Ludeña, P. Vashishta and R. F. Bishop (Nova Science, Commack, New York, 1996), p. 91.

27. C. Zeng, D. J. J. Farnell and R. F. Bishop, J. Stat. Phys. 90, 327 (1998).

28. R. F. Bishop, D. J. J. Farnell and J. B. Parkinson, Phys. Rev. B 58, 6394 (1998).

29. J. Rosenfeld, N. E. Ligterink and R. F. Bishop, Phys. Rev. B 60, 4030 (1999).

30. R. F. Bishop, D. J. J. Farnell, S. E. Krüger, J. B. Parkinson, J. Richter and C. Zeng, J. Phys.: Condens. Matter 12, 7601 (2000).

31. R. F. Bishop, D. J. J. Farnell and M. L. Ristig, Int. J. Mod. Phys. B 14, 1517 (2000).

32. S. E. Krüger, J. Richter, J. Schulenberg, D. J. J. Farnell and R. F. Bishop, Phys. Rev. $B$ 61, 14607 (2000).

33. D. J. J. Farnell, R. F. Bishop and K. A. Gernoth, Phys. Rev. B 63, 220402R (2001).

34. D. J. J. Farnell, K. A. Gernoth and R. F. Bishop, Phys. Rev. B 64, 172409 (2001).

35. D. J. J. Farnell, K. A. Gernoth and R. F. Bishop, J. Stat. Phys. 108, 401 (2002).

36. N. B. Ivanov, J. Richter and D. J. J. Farnell, Phys. Rev. B 66, 014421 (2002).

37. D. J. J. Farnell and R. F. Bishop, High-order coupled cluster method (CCM) calculations via parallel processing: An application to the kagome antiferromagnet, http://arxiv.org/abs/cond-mat/0311126.

38. S. E. Krüger, D. J. J. Farnell and J. Richter, Int. J. Mod. Phys. B 17, 5347 (2003).

39. R. Darradi, J. Richter and D. J. J. Farnell, J. Phys.: Condens. Matter 17, 341 (2005).

40. R. Darradi, J. Richter and D. J. J. Farnell, Phys. Rev. B. 72, 104425 (2005).

41. D. J. J. Farnell, J. Schulenberg, J. Richter and K. A. Gernoth, Phys. Rev. B 72, 172408 (2005).

42. S. Krüger, R. Darradi, J. Richter and D. J. J. Farnell, Phys. Rev. B 73, 094404 (2006).

43. D. J. J. Farnell and R. F. Bishop, The coupled cluster method applied to the XXZ model on the square lattice, http://arxiv.org/abs/cond-mat/0606060.

44. A. W. Sandvik, Phys. Rev. B 56, 11678 (1997).

45. W. Zeng, J. Oitmaa and C. J. Hamer, Phys. Rev. B 43, 8321 (1991).

46. C. J. Hamer, Z. Weihong and P. Arndt, Phys. Rev. B 46, 6276 (1992).

47. J. Richter, J. Schulenburg and A. Honecker, in Lecture Notes in Physics, Vol. 645, eds. U. Schollwöck, J. Richter, D. J. J. Farnell and R. F. Bishop (Springer-Verlag, Berlin, Heidelberg, 2004), pp. 85-153.

48. J. D. Reger, J. A. Riera and A. P. Young, J. Phys.: Condens. Matter 1, 1855 (1989).

49. W. H. Zheng, J. Oitmaa and C. J. Hamer, Phys. Rev. B 44, 11869 (1991).

50. L. Capriotti, A. E. Trumper and S. Sorella, Phys. Rev. Lett. 82, 3899 (1999).

51. R. P. Singh and D. A. Huse, Phys. Rev. Lett. 68, 1766 (1992).

52. S. J. Miyake, J. Phys. Soc. Jpn. 61, 983 (1992).

53. A GPL licensed version of the CCM code is available online at: http://wase.urz.unimagdeburg.de/jschulen/ccm/.

54. P. M. Morse and H. Feshbach, in Methods of Theoretical Physics, Part II. (McGrawHill Book Company, New York, 1953). 